\title{
Environmental and infectious conditions in sports
}

\author{
Thomas S. Weber, MD ${ }^{\mathrm{a}, \mathrm{b}, *}$ \\ ${ }^{a}$ Department of Family Medicine, The Ohio State University, Columbus, OH 43210, USA \\ ${ }^{\mathrm{b}}$ Sports Medicine Center, 2050 Kenny Road, Columbus, OH 43221, USA
}

\section{Cardiopulmonary function in athletes}

In order for an athlete to reach peak performance, many variables must be optimized. Daily training focuses on muscle strengthening, sharpening fine-motor skills and increasing endurance. Because the heart and lungs are the true "engine" of the athlete, much time and attention are given to the cardiopulmonary system during training sessions to maximize performance. Even the slightest change in cardiac output or ventilatory function can have dramatic effects on how well the athlete is able to compete. Environmental factors on playing fields and in arenas can negatively influence performance, as can a variety of infectious agents passed by teammates, competitors, or family members. Below is a review of the common infections and environmental conditions encountered in the care of athletes.

For purposes of review, the cardiovascular system is a closed network of arteries, arterioles, capillaries, venules, and veins, all circulating nutrient-rich blood via the pumping of the heart. Oxygen is delivered to the brain and skeletal muscle while the waste products of carbon dioxide and lactic acid are cleared. Cardiac output (CO) is a standard measurement of the volume of blood provided to the systemic circulation per minute and is determined by the product of the heart rate $(\mathrm{HR})$ per minute and the stroke volume $(\mathrm{SV} ; \mathrm{CO}=\mathrm{HR} \times \mathrm{SV})$. An athlete's cardiac output is regulated and influenced by a multitude of factors, including systemic venous resistance, ventricular compliance, after-load pressures, and intravascular blood volumes. Fever, dehydration, and environmental and body temperatures can all alter how efficiently the heart distributes blood to the tissues.

An athlete's heart responds to endurance training with myocardial hypertrophy that results in a larger stoke volume and lower heart rate. The left ventricular wall

* Sports Medicine Center, 2050 Kenny Road, Columbus, OH 43221.

E-mail address: tweber@ah.com 
thickness increases, yet compliance does not change substantially, allowing for appropriate distention of the chamber and ease of filling during diastole. Several conditions mimic this normal process, but if wall thickness increases too much, hypertrophy then becomes pathologic and cardiac function is impaired. The Starling curve accurately depicts the balance between stroke volume and filling pressures, as seen in Fig. 1. If left-ventricular end diastolic pressure increases and stretches the myocardial fibers beyond their normal capacity, cardiac output falls off rapidly. Diseased myocardium loses its compliance and inherent ability to react to different filling pressures and volumes, which then results in a very narrow physiologic window for proper cardiac output.

The pulmonary system is also highly regulated by numerous intrinsic and extrinsic factors. Respiratory rate, tidal volumes, and expiratory flow rates are all subject to change according to a variety of influences, including air temperature, weather conditions, degree of exertion, and the presence of inflammatory reactions. Air pollution, bronchial reactivity, extremes of temperature, and high altitude are just some of the factors with which athletes must contend. Pulmonary function testing evaluates the lungs' capacities and flow rates at rest and with exercise. Often, environmental conditions may be hard to reproduce in the lab, so field testing may be necessary to accurately measure how well the pulmonary system is performing.

Pulmonary function is also included in determining the aerobic capacity $\left(\mathrm{VO}_{2} \max \right)$ of an athlete. This quantifies how much oxygen in milliliters is used in one minute per kilogram of body weight. Obviously, optimal pulmonary function is crucial to the efficient diffusion of oxygen from the atmosphere across the alveolar wall into the capillary. Nasopharyngeal congestion, bronchial edema, or smooth muscle hypertrophy can limit the amount of oxygen that is delivered to the alveoli. The cardiovascular system's transport and the muscles' ability to use delivered oxygen also factor into the $\mathrm{VO}_{2} \max$ determination. As an athlete's conditioning improves, so too does her $\mathrm{VO}_{2} \mathrm{max}$, up to her highest genetic potential. When $\mathrm{VO}_{2}$ max levels fall, athletic performance will subsequently decline as well. Deciphering which part of this physiologic cascade is problem-

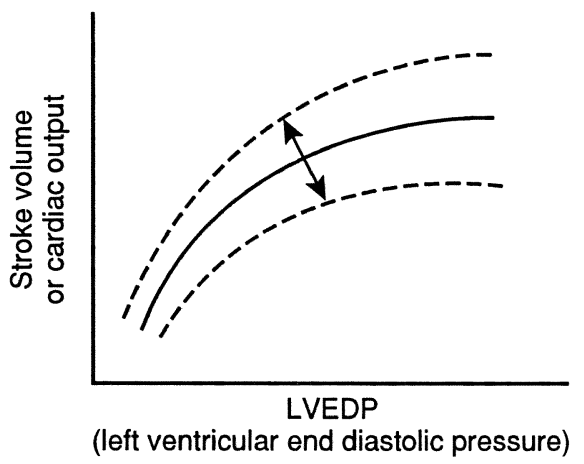

Fig. 1. The Starling Curve. 
atic can be a diagnostic challenge, but is crucial to restoration of full function in the athlete.

\section{Infection and the immune response}

Is the competitive athlete more susceptible to infections than the average population? Do ill athletes actually perform less well than their healthy counterparts? There is an ongoing debate over the questions surrounding exercise's harmful or beneficial effects on immune function, with both sides providing wellconducted studies stating their case. Certain physiologic changes unarguably occur when the athlete becomes ill or run-down. Fever is a natural response to infection, and triggers a cascade of events that change cardiopulmonary performance. Fever stimulates vasodilatation, which decreases systemic venous return, limiting preload filling pressures and eventually leading to a decrease in cardiac output. The heart must then increase its rate to make up for the diminished volume. Increased respiratory rates develop to compensate for the increased temperature and the increased demand for oxygen to active inflammatory cells. Fever is also responsible for immune-mediator activation via neutrophils, lymphoctyes, and cytokines. These cells cause increased capillary permeability, which allows the host defense system access to the tissues where the infection is occurring. This process can lead to bronchial mucosal edema and shifts in extracellular fluid levels, both of which compromise the ability to deliver oxygen to the athlete's skeletal muscle.

Dehydration can be the result of increased core temperature and respiratory rate from fever, or it can be the direct result of vomiting and diarrhea from gastrointestinal infections. The athlete's body depends on an appropriate hydration level not only to deliver nutrients to the muscles, but also to aid in core-cooling processes such as sweating. Suboptimal hydration can result in muscle tetany (cramping), hyperthermia, and decreased cardiac output and $\mathrm{VO}_{2}$ max. When athletes are ill, it is crucial that they increase not only their fluid intake but also their general caloric intake as well, because the activated immune system increases caloric expenditures well above resting states. There is an inverse relationship between plasma glucose levels and activation of stress hormones such as cortisol. One theory postulates that maintaining higher plasma glucose levels through the consumption of carbohydrate drinks during prolonged exertion decreases cortisol levels and in turn, places less demand on the immune system [1].

Numerous studies have shown the effects that moderate exercise has in reducing the incidence of simple upper respiratory tract infections (URTIs). Previously sedentary women were randomized to near-daily walking or remaining sedentary for 3 to 4 months. The active group reported half as many days with URTI symptoms. Brisk walking for 30 to 45 minutes at least five times per week showed the greatest decrease in symptoms [2,3]. Moderate exercise is believed to stimulate the function of natural killer cells; however, strenuous activity may actually suppress neutrophil function [4,5]. Increased minute ventilation with 
Table 1

Immune system changes after heavy exertion [6]

\begin{tabular}{lll}
\hline Change & Mechanism & Possible result \\
\hline Increased neutrophils & Increased cortisol, GH & Bacterial protection \\
Increased phagocytosis & Granulocytes/monocytes & Bacterial clearance \\
Decrease ciliary clearance & Nasal/mucous drying & Failed particle clearance \\
Decrease natural killer cells & Unknown & Viral susceptibility \\
Decrease T-cell function & Decreased lymphocytes & Viral susceptibility \\
Decrease IgA concentration & Salivary/nasal levels & Reduced immunity \\
Blunted MHC expression & Unknown & Decreased antigen presentation
\end{tabular}

From Nieman DC. Is infection risk linked to workload? Med Sci Sports Exer 2000;32(Suppl 7): S406-11; with permission.

exercise may help ciliary function in clearing pathogens from the nasotracheal and bronchial tissue. The magnitude of change in the immune system during actual exercise is largely unknown or quantified, but many studies have failed to show large differences in certain immune levels in the postexercise resting state between athletes and nonathletes [6]. Therefore, the duration of these exercise effects on the immune system is unknown. Strenuous exercise, however, appears to be different from mild to moderate levels. Marathoners and triathletes may be particularly susceptible to infectious agents during a window of time following their training or competition. Table 1 lists the transient effects recorded after strenuous exercise.

Other investigations have sought to make a connection between glutamine levels and the immune system. Glutamine is a known precursor to the creation of some immune cell lines. Studies revealed a decline in this amino acid during exercise, and it was postulated that this may be a cause for the increased risk of viral infections. Subsequent glutamine supplementation studies have given no clinically significant results in boosting the immune systems of athletes [7,8,]. Therefore, until we perfect our assay analysis techniques and truly understand the intricacies of the immune system, it will be hard to conclusively say how much exercise is safe for the immune system and how long it is run down after bouts of heavy exertion. For now, it appears that regular, moderate-intensity exercise can be beneficial to the immune status. Those participating in more strenuous events should realize the potential for infection and make it a priority to rest sufficiently after training and competition, and to replenish energy stores, hydration levels, and vitamins.

\section{Infectious conditions of the lungs and respiratory tract}

Upper respiratory tract symptoms of cough, rhinorrhea, or sore throat are by far the most common infectious complaints of athletes at the time of an event, both recreational and professional. The incidence varies by season, age of the athlete, climate, and level of exertion. More serious infections such as bronchitis and pneumonia may begin with symptoms similar to a URTI. Most current 
literature demonstrates the athlete's susceptibility to viral but not bacterial infections. As mentioned previously, there is good evidence that supports the belief that mild to moderate exercise has positive effects on the immune system's ability to ward off infection. Because of the relatively high prevalence of URTI, much research has focused on different ways to prevent these common ailments, which can affect performance or delay participation for days and weeks.

The common cold lasts 5 to 10 days and its symptoms are generally mild enough so as not to prevent the athlete from continuing training or competing. Rhinoviruses, coronaviruses, respiratory syncytial virus (RSV), parainfluenza viruses, and adenoviruses are the usual perpetrators, though rarely is testing done in a clinical setting to identify which of these is actively infecting the patient. Simple decongestants and cough suppressants often provide adequate relief of symptoms. Care must be taken to review the lists of banned substances in collegiate, professional, and Olympic sports before prescribing them. Weidner et al demonstrated the effects of a URTI caused by rhinovirus 16. A wellconducted trial showed no significant difference between those inoculated with the virus versus those receiving placebo at days 2, 5, and 8 in pulmonary function, $\mathrm{VO}_{2} \max$, and exercise to fatigue [9]. This supports the concept that there are few physiologic changes in the athlete's body during an uncomplicated URTI, and athletes should be considered medically appropriate for return to participation even while symptomatic.

It is unclear if excessive exercise, such as running a marathon while symptomatic with a URTI, predisposes an athlete to a prolonged course of illness or progression to a more serious infection such as pneumonia. The true incidence of URTI in these populations is heavily varied. Nieman et al found only one of seven marathoners studied complained of URTI symptoms after completing a 1987 Los Angeles marathon [10]. Peters et al found a threefold increase in URTI symptoms in runners if they completed an ultramarathon in under 4 hours compared with baseline controls, and found only a small increase in those taking 5 to 6 hours to complete the race [11]. Future research will attempt to find ways to combat these common infections. One such attempt studied vitamin $\mathrm{C}$ at doses of $600 \mathrm{mg}$ per day for 3 weeks following a marathon, which decreased the incidence of URTIs by $50 \%$ in those taking it [12]. Similar studies, however, have not shown a benefit [13].

Bronchitis and pneumonia, more significant respiratory processes, can be much more problematic to an athlete's training schedule or performance on game day. Bronchial inflammation and edema from infection often mimic symptoms experienced by asthmatics, with a prolonged expiratory phase in the respiratory cycle, as measured by the forced expiratory volume in one second (FEV1). A productive cough, audible wheezing, or difficulty catching the breath can signal bronchial infections. The distribution of pathogens appears to favor viral infections such as influenza, but also includes bacterial species such as Moraxella catarrhalis, Haemophilus influenza or Streptococcus pneumoniae. Although determining the presence of a bacterial process definitively is difficult at best, treatment often consists of antibiotics such as macrolides (erythromycin, clari- 
Table 2

Causative agents of pneumonia

\begin{tabular}{ll}
\hline Atypical pneumonia & Lobar pneumonia \\
\hline Mycoplasma Pneumoniae & Streptococcus pneumoniae \\
Chlamydia pneumoniae & Moraxella catarrhalis \\
Legionella pneumophila & Haemophilus influenzae \\
Chlamydia psittaci & Staphylococcus aureus \\
Influenza A, B & Oral anaerobes \\
RSV & Pseudomonas aeruginosa \\
Pneumocysitis carinii & E. Coli or Klebsiella pneumoniae
\end{tabular}

thromycin, azithromycin) or doxycycline. Bronchodilators or inhaled steroids may be necessary to provide some relief for the athlete who continues to train. Resolution of symptoms is often prolonged, and some physicians may treat the patients with oral steroids in an attempt to curtail the postinfectious inflammation that often follows the acute bronchial infection.

Lobar pneumonia and atypical pneumonia are generally more serious infections that require some time off from training and competition. High fevers, lethargy, and muscle aches often coexist, with or without a productive cough. Atypical or community-acquired pneumonia is more common in athletes below the age of 40 . Table 2 lists the organisms responsible for infection. Empyema, hypoxia, and sepsis are unusual complications and are rarely seen with atypical agents.

Treatment involves antibiotics in suspected or documented cases confirmed with a chest radiograph. Again, doxycycline and macrolides for 14 to 21 days will effectively treat the majority of cases. Resistant strains are prevalent in some areas and fluoroquinolones can be effective in those situations. Care is taken to avoid giving these to pediatric athletes or the skeletally immature, as they are known to have an increased risk of osteochondrosis. Pneumocystis carinii and Mycobacterium tuberculi have made a resurgence with the increased number of immunosuppressed patients, so the absence of response to typical treatment warrants further investigation. Return to play should be guided by the physician based upon the gradual disappearance of symptoms, with caution not to advance the athlete too quickly back into strenuous training. Typically, one full week of rest and a graded exercise program thereafter afford appropriate time for the immune system response [14]. As in bronchitis, some postinfectious inflammatory effects may be seen, and full return to play may take more than one month.

\section{Infectious conditions of the heart}

Fortunately, for most athletes, a cough and runny nose are the worst infectious manifestations that they will encounter. It must always be remembered, however, that a "simple" viral syndrome can turn into a life-threatening illness by attacking heart structures directly or by creating immune cross-reactivity with cardiac myocytes. The true incidence of cardiac infection in athletes is unknown, but because of its possible devastating effects, physicians and trainers must 
remain alert for any signs or symptoms suggestive of cardiac involvement with any type of ailment. Unfortunately, those signs are often nonspecific and can be very mild. Chest pain with or without radiation, new onset of dyspnea with exercise, or changes in heart rate or blood pressure following a systemic illness should signal the need to perform appropriate testing. Obscure symptoms of prolonged fatigue or low-grade temperatures may also suggest an indolent infection in the myocardium.

Myocarditis describes the inflammatory state of the myocardium, as evidenced in the clinical changes on both electrocardiogram (ECG) and at the cellular level on myocardial biopsy. Symptoms at the onset may include the following: fatigue, fluid retention, fever, or palpitations. Signs may include tachycardia or irregular rhythms, or an S3 heart sound may be appreciated on auscultation. White-blood-cell count and erythrocyte sedimentation rate may be elevated in the serum, though absence of either does not rule out the disease. ECG changes may include sinus tachycardia or subtle, nonspecific ST segment wave changes. This can be misleading in athletes who display traits characteristic of the athletic heart. Normal ECG findings with these patients may show sinus arrhythmia, junctional escape beats, first-degree AV block or second-degree block (Mobitz type I), increased voltage, or increased QT intervals [15]. Thus, differentiating between a normal athletic heart and the pathologic heart of myocarditis can be difficult. Though invasive, myocardial biopsy may be necessary to make the diagnosis. Plain chest radiograph may be unremarkable or may reveal cardiomegaly and pulmonary congestion. Echocardiograms may show akinetic wall-motion abnormalities and evidence of ventricular hypertrophy, dilation, or decreased ejection fraction.

The pathogenesis of myocarditis has been studied extensively in animal models and appears to be two-staged. Initially, during the infectious stage, viral replication occurring in the myocytes may cause mild damage and typically lasts 7 to 14 days. Listed in Table 3 are some of the most commonly identified organisms implicated in myocarditis. Viruses are the most common cause in the United States [16]; however, often no pathologic agents are identified by serology or biopsy during or after the initial illness.

Often this first stage is all that occurs, and the infection may pass unnoticed. It is the second stage where more severe destruction can occur as the virus is being cleared. A T-helper cell-directed, lymphocytic response against the myocardial/ viral antigen mistakenly destroys myocardial cells, resulting in tissue necrosis,

Table 3

Some common infectious causes of myocarditis

\begin{tabular}{llll}
\hline Viral & Bacterial & Fungal & Rickettsial \\
\hline Coxsackie B, A & Salmonella typhi & Candidiasis & RMSF \\
Influenza B, A & Staph, Strep & Histoplasmosis & Q fever \\
Echovirus, HIV & Clostridia & Aspergillosis & Typhus \\
CMV, EBV & Gonococcus & Blastomycosis & \\
\hline
\end{tabular}


edema, and eventual scar tissue formation [17]. If enough myocytes are damaged, the ventricles in particular lose their inherent strength and congestive heart failure may ensue.

The dangers for the athlete who develops myocarditis are twofold. The shortterm fear lies in the fact that as myocytes are destroyed and scar tissue is laid down, an arrythmogenic substrate is created. As with myocardium destroyed by ischemia, this tissue may conduct electrical currents at different velocities from healthy cells and result in a variety of arrhythmias, from atrial premature complexes to ventricular tachycardia or fibrillation. All athletes with suspected or confirmed myocarditis must stop exercising because of the inherent increased risk for malignant rhythms and sudden cardiac death. The length of time that these athletes or patients are at risk for developing arrhythmias is unknown.

Congestive heart failure is the other most significant complication from this infection. Diuretics and angiotension conversion enzyme (ACE)-inhibitors may be used to afford symptomatic relief, but it is not known if they offer any slowing of progression or reversal of structural damage. Some patients may recover spontaneously, whereas others will have permanent cardiac dysfunction. Some authors suggest at least a six-month period of no exercise, then re-examining for symptoms, evidence of relevant arrhythmias, and level of ventricular function before considering return to play [18].

The pericardium is also susceptible to inflammation from infectious and other irritant causes. Though generally much less severe than myocarditis, pericarditis can have serious complications as well. Most cases are classified as idiopathic, but the majority of cases are likely viral. Coxsackie, adeno- and echoviruses are common causes, but pericarditis may arise from bacterial infection, medications, local radiation, collagen-vascular disorders, or autoimmune diseases. Patients present with a complaint of substernal chest pain following a prodromal syndrome suggestive of a viral infection. Pain is classically worsened with supine position and deep inhalations and is often lessened by sitting upright and leaning forward, and deep inhalations.

The electrocardiogram is likely the most helpful tool in making the diagnosis, and it is crucial to rule out cardiac ischemia, because it often presents with similar symptoms. Four stages of ECG changes can occur with the natural progression of pericarditis. Stage I shows an elevated ST segment and upright T waves, often across multiple precordial leads. In stage II, the ST elevation returns to normal and the $\mathrm{T}$ wave becomes upright flat. The $\mathrm{T}$ wave becomes inverted in stage III and returns to near normal in stage IV [19] erythrocyte sedimentation rate (ESR) and leukocytes may be elevated transiently. Treatment consists of rest for the athlete until he is symptom free and the ECG returns to normal. Most viral or idiopathic cases respond well to nonsteroidal anti-inflammatories. The most serious complications are rare in otherwise healthy individuals, but include pericardial effusion with or without tamponade. If the effusion is large enough or develops rapidly enough, its pressure on the ventricle can lead to rapid cardiac decompensation. It is best diagnosed by echocardiogram and requires immediate drainage by pericardiocentesis. Constrictive pericarditis can also develop as a 
result of the acute inflammation. This leads to thickened, noncompliant pericardium, which does not allow proper filling during diastole and results in decreased cardiac output. Patients with these complications may never be able to return to the playing fields of their sports. Formal cardiology consultation for athletic clearance is essential.

Lastly, infectious endocarditis is another rarity in the athletic population, largely afflicting intravenous drug users and those with congenital anomalies of the heart and its valves. With the prevalence of injected anabolic steroid and growth hormone use, however, not all athletes are free of risk. Along with investigating for appropriate risk factors, symptoms such as fever, lethargy, or malaise should be investigated. Diagnostic signs include new onset of a heart murmur, petechiae, splinter hemorrhages, Osler nodes, Janeway lesions, or Roth spots on the fundi, depending on the duration of the disease course. Major criteria for the diagnosis are positive blood cultures for typical organisms - Streptococcus viridans and bovi, Enterococcus, and Staphylococcus, or any from the HACEK group (Haemophilus, Actinobacillus, Cardiobacterium, Eikenella, or Kingella) [20] - or an oscillating mass/abscess on a heart valve, as demonstrated on echocardiography. Minor criteria include fever $>38.0 \mathrm{C}$, predisposing risk factors, vascular phenomena, immunologic phenomena, or echocardiographic evidence. Treatment consists of long- term antibiotics and possible surgery for abscess removal if present. Mortality is nearly $25 \%$ in the acute setting, and longterm complications include scarring of affected valves, recurrence of infection, and heart failure [21].

\section{Environmental conditions affecting the pulmonary system}

Fortunately the heart is protected from most external factors by a costal shield and thick pectoralis muscles. The lungs, however, are in constant contact with the outside world with each breath. A majority of athletes compete outdoors in urban areas where levels of ozone, carbon monoxide, sulfur dioxide, and nitrogen oxides are elevated. Cough, shortness of breath, or wheezing may develop in nonasthmatic competitors as these chemical irritants stimulate the immune system. Athletes also compete in rural areas where cars and smog are nowhere to be found. Atmospheric pressures and oxygen concentrations vary for sports enthusiasts as they climb mountains or swim to the depths of the ocean floor. Again, it is primarily the pulmonary system that must adapt to these changes.

The burning of fossil fuels in combustion engines of cars and trucks, factories, and utilities is responsible for the production of most of the photochemical pollutants we encounter on a daily basis. Ozone $\left(\mathrm{O}_{3}\right)$ is a free-radical product of oxygen $\left(\mathrm{O}_{2}\right)$, and is found in higher levels near major urban areas. Long-term effects of ozone on the body are unknown, but the effects of free-radicals on the body have been linked to cancer. Ozone concentration in the atmosphere is measured in parts per million (ppm) and cities like Los Angeles have levels of between 0.15 and $0.35 \mathrm{ppm}$. Adams et al studied 10 male distance runners and 
exposed them to varying levels of $\mathrm{O}_{3}$, then measured their forced vital capacity (FVC) and their FEV1. They found significant decreases in FVC and FEV1 when the runners were exposed to levels of both 0.20 and $0.35 \mathrm{ppm} \mathrm{O}_{3}$ while exercising as compared with no ozone exposure [22]. It is important to remember that in the majority of these studies, the athletes studied were not asthmatic at baseline, and the results seen would likely be further amplified in those with established reactive airway disease.

Another group looked at seven male and female cyclists who were exposed to $0.21 \mathrm{ppm}_{3}$ during a one-hour training session, and again found significant decreases in FVC compared with baseline. The subjects also complained of symptoms of upper airway irritation, along with cough and chest tightness [23]. In addition, higher ambient temperatures seem to increase the subjective effects of ozone. Interestingly, there may be some tolerance that the lungs develop to ozone after periods of longer exposure. Residents of Los Angeles showed a minimal response to $0.4 \mathrm{ppm}$ of ozone, whereas a group studied in Montreal had significant reactions when exposed to that same level [24]. Another 14 subjects were exposed to $0.4 \mathrm{ppm}$ levels of $\mathrm{O}_{3}$ for 5 straight days for 3 hours each day. They developed significant decreases in their FVC for the first 3 days, then those changes dissipated [25]. It is unclear how rapidly the body loses these adaptive tolerances, but this may have clinical applications for athletes. If time permits, arriving at the site of a competition one week before endurance activities where minute ventilation will be high for extended periods of time may be beneficial for adaptation.

Sulfur dioxide $\left(\mathrm{SO}_{2}\right)$ is another common air pollutant arising from coal and wood fires. A water-soluble compound, $\mathrm{SO}_{2}$ is mostly absorbed in the nasopharynx while breathing at rest, and less than $1 \%$ even makes it to the oropharynx [26]. In athletes, however, high ventilatory rates lead to drying of the mucous membranes, and thus the potential for inhalation increases. A level of 1 to $2 \mathrm{ppm}$ appears to be the threshold for causing increased respiratory resistance in healthy individuals [27]. Koenig et al found a 3 to 22 fold increase in pulmonary functional changes when athletes were exercised and exposed to $1.0 \mathrm{ppm}$ of $\mathrm{SO}_{2}$, as compared with exposure during rest [28]. Bell et al, however, failed to demonstrate any significant changes in pulmonary function in exercising patients when exposed to levels of $1.0 \mathrm{ppm}$. They did, however, reproduce the inflammatory effect of ozone in the same study [29].

Carbon monoxide $(\mathrm{CO})$ and nitrogen dioxide $\left(\mathrm{NO}_{2}\right)$ are two additional common compounds humans are exposed to daily. $\mathrm{NO}_{2}$ alone does not seem to produce any measurable effects on lung function using standard methods, but questions remain regarding its additive effects with increased ozone concentrations [30]. Carbon monoxide exerts its effects by binding to hemoglobin with an affinity 210 times greater than that of oxygen. This decreases oxygen-carrying capacity and decreases the release of oxygen to the tissues (it shifts the $\mathrm{O}_{2}$ dissociation curve to the left). People who train near heavy automobile traffic could be exposed to levels of $\mathrm{CO}$ between 10 and 50 ppm. Raven and colleagues observed a linear decrease in $\mathrm{VO}_{2}$ max as carbon monoxide levels increase in the blood [31]. Therefore, a runner along a busy freeway may develop a carboxy- 
hemoglobin level of $7 \%$ after only 1 hour, and hence reduce his $\mathrm{VO}_{2}$ max capability by the same amount [31,32]. Patients often remain symptom-free until levels reach $20 \%$ to $30 \%$.

With the world's population exploding, the levels of these common pollutants are likely to continue to rise in the next several decades. Athletes are forced to contend with these ambient conditions. Filters or masks are cumbersome and may not be small enough to trap particles less than 3 to 4 microns in size while still allowing the athlete to take deep breaths. Bronchodilators and inhaled antiinflammatories may become more common in nonasthmatic competitors if these photochemicals continue to plague our environment. Recommending that our athletes exercise away from cities and automobiles is worthwhile but impractical. Additional study of training environments and the possible additive effects of pollutants on pulmonary function are necessary before more definitive recommendations can be made.

\section{High-altitude effects on the pulmonary system}

Even though the air may be cleaner high up in the Rockies, it is also considerably thinner. Up to a certain height, oxygen concentrations are similar to those at sea level, but the lower oxygen levels and lower partial pressure of oxygen at high altitudes cause active people to be short of breath. The practice of living and training at high altitude in order to boost performance at sea level has been around for years, but the effects seem to vary somewhat. New studies evaluate this style of training from a different angle. Mountain climbers and extreme athletes expose themselves to even tougher conditions as they explore and compete near the top of the world. Gradual exposure to the thinner air usually is without complication, but ascending and exerting too rapidly may not allow the lungs to adjust quickly enough. High-altitude pulmonary edema (HAPE) is a topic receiving considerable attention, as its incidence has risen with the increased number of people making their way to the summit.

At higher altitudes, the body responds to decreased oxygen saturation by releasing erythropoetin (EPO) from renal cells, which stimulates the production of erythrocytes to bind whatever oxygen is available. The concentration of $2,3^{-}$ biphosphoglyceric acid(2,3-BPG) also aids in lowering the oxygen tension, and fosters oxygen release from hemoglobin into the tissues. Living and training at high altitude has proven to benefit subsequent performance at sea level, but that edge over the competition is quickly lost over the course of 2 to 3 weeks. One group of experts recommends that the ideal training circumstance is to train for 4 weeks at 2200 meters ( 7000ft) [33]. Baker and Hopkins summarized 17 studies that evaluated altitude training for improvement in sea-level $\mathrm{VO}_{2}$ max and found 5 studies showing a definite positive effect, 1 showing a definite negative effect, and the rest showing effects somewhere in between [34]. Study quality was not consistent throughout, however, as there was wide variability in the number of subjects, duration of time, and elevation levels. 
Stray-Gundersen, Chapman, and Levine studied elite runners who lived at 2500 meters for 4 weeks while performing intense non-daily workouts at 1250 meters. There was no control group, but the studied athletes showed significant improvements in their 3000-meter race times. $\mathrm{VO}_{2}$ max was increased by $3 \%$ from baseline and EPO levels doubled [35]. Altitude acclimation is essential to allow the body time to adapt and recover from the strain of lower oxygenation on the cardiopulmonary system. These effects are acquired and transient in most individuals. Only athletes born and raised at higher altitudes appear to retain any long-term benefits of such altitude exposure.

Rapid ascents into high altitudes are generally tolerated poorly by both athletes and nonathletes alike. Even those people born at high altitudes need appropriate time to respond to such low-oxygen environments. In order to supply the body with increased levels of oxygen when the available resources are low, respiratory rates must increase. Increased respiratory rates will decrease carbon dioxide levels, often resulting in a respiratory alkalosis, which is responsible for many of the symptoms of acute mountain sickness(AMS). Headache, nausea, fatigue, and insomnia can appear as early as several hours after ascent to 2000 to 2500 meters. This syndrome is typically short lived and remedied with rest, proper hydration, or supplemental oxygen. Severe cases may need all of the above and descent to lower altitude. Acetozolamide, a carbonic anhydrase inhibitor, facilitates the release of bicarbonate in the kidneys and produces a $74 \%$ improvement in symptoms compared with placebo [36]. Dexamethasone has shown similar results [37].

Climbers, skiers, or visitors to the mountains who complain of cough, wheezing, or shortness of breath must be evaluated for HAPE, a noncardiogenic form of pulmonary edema that may be fatal if left untreated. Incidence worldwide varies, but was estimated at $0.01 \%$ to $0.1 \%$ of visitors in the late 1970 s to early 1980s at the Colorado ski resorts [38]. It is rarely seen below 2500 meters and typically presents 1 or 2 days after reaching a new altitude, but does not usually occur after spending 4 days at the same height [39]. Low-grade fever or rales may be present and pre-existing URTIs or bronchitis may put people at a higher risk for developing HAPE, especially children [40]. Chest radiographs and computed tomography (CT) scans often reveal a patchy infiltrate pattern. Only late in the disease course does pink, frothy sputum or frank hemoptysis develop. Its pathophysiology appears to be related to altered perfusion, from vasoconstriction in pulmonary capillaries in response to diminished oxygen levels. Pulmonary hypertension ensues, as does elevated capillary wedge pressure, which some researchers believe is the cause of the pulmonary edema [41]. Those who develop HAPE once have a much higher rate of recurrence on subsequent trips.

Treatment involves descent and supplemental oxygen, as with AMS. In severe cases or if descent is not immediately possible, nifedipine has proven to be beneficial for relieving symptoms by reducing pulmonary artery pressures by $30 \%$. It has also been proven to decrease the incidence of HAPE recurrence in susceptible climbers [42]. Some experts recommend ascending no more than 300 to 350 meters per day as a means of preventing altitude-related conditions. 
Cremona and colleagues studied 262 consecutive climbers of a 4559-meter peak, both before ascent (at 1200 meters) and one hour after arrival at the summit ( $\sim 15 \mathrm{hrs}$ later). Evaluations included chest radiographs, lung auscultation, oximetry, spirometry, carbon monoxide transfer, and closing volume. Interestingly, they found that $15 \%$ of the subjects had chest rales or interstitial edema on chest radiograph. Of those without edema, $74 \%$ had increased closing volumes, suggestive of subclinical pulmonary edema. Only one climber was evacuated for HAPE. They proposed that larger lung size at baseline is protective against HAPE because of intrinsically lower vascular resistance associated with larger lung cross-sectional area [43]. This study suggests that a much higher incidence of pulmonary edema may exist with rapid ascent than was previously thought.

\section{Decompression illness in divers}

Athletes who explore the world under the ocean face a different set of risks and dangers in their sport. At sea level, the body is subject to ambient pressures measured at 1 atmospheric absolute of pressure (ATA). As the diver descends into the water, these pressures double at 33 feet, triple at 66 feet, and so on. Fluidfilled organs are not affected, but air-filled organs such as the middle ear and the lungs are susceptible to pressure-related injury, so-called barotrauma. With descent, gases such as nitrogen become soluble and dissolve into a liquid state, whereas with ascent, solubility decreases and they return to their gaseous state. Ascending too quickly causes these gases to precipitate into bubbles, which can be harmful to the closed network of the cardiovascular system. Collectively, these changes result in decompression sickness (the bends).

Pain in the joints from the accumulation of nitrogen bubbles is the most common clinical symptom seen in divers. Bubbles in the skin or lymph system can develop as well, and these manifestations are known as type I decompression sickness. When the heart, lungs, or nervous system are affected, it is classified as type II. With improper ascent, arterial gas embolisms may occur from ruptured alveoli secondary to barotrauma (known as pulmonary overinflation syndrome) [44]. Improper ascent occurs when the diver rises too quickly or fails to exhale on ascent. After alveolar rupture, bubbles may enter the pulmonary venous system and then travel through the left atrium and into the systemic arterial circulation. These bubbles may then travel to the brain or spinal cord, causing cerebral vascular accidents, or rarely to the coronary arteries, causing myocardial infarction. In recreational divers, neurologic events comprise $80 \%$ of reported cases of decompression problems. Those divers with decompression illness without a history of a faulty ascent should be screened for a patent foramen ovale. This right-to-left shunt may allow venous bubbles access to the arterial circulation [45].

Treatment of decompression illness focuses on returning the diver to a higherpressure state, hoping to once again dissolve the nitrogen bubbles into liquid. This is accomplished with a hyperbaric oxygen chamber, which shrinks the bubbles to prevent further damage and supplies high oxygen levels to ischemic 
tissues. In addition, fluids are recommended either orally or intravenously. Steroids may be useful if neurologic damage is suspected. Diazepam can help minimize vertigo and nausea if there is vestibular damage to the ears [46]. Length of treatment and the severity and duration of symptoms dictate levels of pressure. Diving is enjoyed by millions of people around the world and is generally uneventful, but there are risks, and the safety of every diver should a priority of the sports medicine practitioner.

\section{Summary}

The hearts and lungs of athletes are subject to damage from a wide array of infections and environmental factors. Mild to moderate exercise has been shown to be beneficial to overall health, and strenuous exercise simply requires proper rest and rehabilitation to ensure its beneficial effects as well. Simple colds and URTIs are very common in athletes and do not usually require significant intervention. Any suspected cardiac infection mandates a thorough evaluation and proper management to prevent catastrophic consequences. High altitudes can be helpful in enhancing performance, but caution must be exercised at even modest altitude to prevent serious complications. With diving, participants should know their time limits and ascend properly to avoid serious complications. Keeping the heart and lungs in a good state of health is a major priority for the weekend warrior and world-class athletes alike. A thorough knowledge of infections and environmental issues in the cardiopulmonary health of athletes should always be of highest priority.

\section{References}

[1] Castell L, Newsholme E. The effects of oral glutamine supplementation on athletes after prolonged, exhaustive exercise. Nutrition 1997;13:738-42.

[2] Nieman D, Nehlsen-Cannarella D, Hensen D, et al. Immune response to exercise training and/or energy restriction in obese women. Med Sci Sports Exerc 1998;30:679-86.

[3] Neiman D, Nehlsen-Cannarella D, Markoff P, et al. The effects of moderate exercise training on natural killer cells and acute upper respiratory tract infections. Int J Sports Med 1990;11: 467-73.

[4] Woods J, Davis J, Smith J. Exercise and cellular innate immune function. Med Sci Sports Exerc 1999;31:57-66.

[5] Pyne D, Baker P, Fricker W, et al. Effects of an intensive 12-week training program by elite swimmers on neutrophil oxidative activity. Med Sci Sports Exerc 1995;27:536-42.

[6] Nieman D. Is infection risk linked to exercise workload? Med Sci Sports Exerc 2000;32(Suppl 7): S406-11.

[7] Kargotich S, Rowbottom D, Keast D, et al. Plasma glutamine changes after high intensity exercise in elite male swimmers. Med Sci Sports Exerc 1996;28:S133.

[8] Keast D, Arstein D, Harper W, et al. Depression of plasma glutamine concentration after exercise stress and its possible influence on the immune system. Med J Aust 1995;162:15-8.

[9] Weidner T, Anderson B, Kaminsky L, et al. Effect of a rhinovirus-caused upper respiratory illness on pulmonary function test and exercise responses. Med Sci Sports Exerc 1997;29(5): $604-9$. 
[10] Nieman D, Johanssen L, Lee J, et al. Infectious episodes in runners before and after the Los Angeles Marathon. J Sports Med Phys Fitness 1990;30:316-28.

[11] Peters E, Bateman E. Ultramarathon running and upper respiratory tract infections. S Afr Med J $1983 ; 64: 582-4$.

[12] Peters E, Goetzsche J, et al. Vitamin C supplementation reduces the incidence of post-race symptoms of upper respiratory tract infection in ultramarathon runners. Am J Clin Nutr 1993;57:170-4.

[13] Himmelstein S, Robergs R, et al. Vitamin C supplementation and upper respiratory tract infections in marathon runners. J Exercise Physiology online 1998;1(2):1-17.

[14] Melham T. Atypical pneumonia in active patients. Phys Sports Med 1997;25(10):43-59.

[15] Holly R, Shaffrath J, Amsterdam E. Electrocardiographic alterations associated with the hearts of athletes. Sports Med New Zeal 1998;25(3):139-48.

[16] Francis G. Viral myocarditis; detection and management. Phys Sports Med 1995;23(7):63-83.

[17] Karjalainen J, Heikkila J, Nieminen M, et al. Etiology of mild acute infectious myocarditis. Acta Med Scand 1983;213:65-73.

[18] Maron B, Isner J, McKenna W. Hypertrophic cardiomyopathy, myocarditis and other myopericardial diseases and mitral valve prolapse. Med Sci Sports Exerc 1994;26(Suppl 10):S261-7.

[19] Vaitkus P, LeWinter M. Pericardial disease. In: Alpert J, editor. Cardiology for the primary care physician. Philadelphia: Current Med. Inc.; 1998. p. 259-66.

[20] Ewy G. Infectious endocarditis. In: Alpert J, editor. Cardiology for the primary care physician. Philadelphia: Current Med. Inc.; 1998. p. 251-8.

[21] Mullant C, Chua Y, Schaff H, et al. Early and late survival after surgical treatment of culturepositive active endocarditis. Mayo Clin Proc 1995;70:517-25.

[22] Adams W, Schelegle E. Ozone and high ventilation effects on pulmonary function and endurance performance. J Appl Physiol 1983;55:805-12.

[23] Folinsbee L, Bedi J, Horvath S. Pulmonary function changes after 1-hour continuous heavy exercise in 0.21ppm ozone. J Appl Physiol 1984;57:984-8.

[24] Hackney J, Linn W, Buckley R, et al. Studies in adaptation to ambient oxidant air pollution: effects of ozone exposure in Los Angeles residents vs. new arrivals. Environ Health Perspect $1976 ; 18: 141-6$.

[25] Farrell B, Kerr H, Kulle T. Adaptation in human subjects to the effects of inhaled ozone after repeated exposure. Am Rev Respir Dis 1979;119:725-30.

[26] Speizer G, Frank R. The uptake and release of $\mathrm{SO} 2$ by the human nose. Arch Environ Health 1966;12:725-8.

[27] Folinsbee L, Bedi J, Horvath S. Pulmonary response to threshold levels of sulfur dioxide (1.0ppm) and ozone (0.3ppm). J Appl Physiol 1985;58:1783-7.

[28] Koenig J, Peirson W, Horike M, et al. Effects of $\mathrm{SO} 2$ plus $\mathrm{NaCl}$ aerosol combined with moderate exercise on pulmonary function in asthmatic adolescents. Environ Res 1981;25:340-8.

[29] Bell K, Linn W, Hazucha M, et al. Respiratory effects of exposure to ozone plus sulfur dioxide in a Southern Californian and Eastern Canadians. Am Ind Hyg Assoc J 1977;38:695-705.

[30] Linn W, Solomon J, Trim S, et al. Effects of exposure to 4-ppm nitrogen oxide in healthy and asthmatic volunteers. Arch Environ Health 1985;40:234-9.

[31] Raven P, Drinkwater B, Ruhling R, et al. Effect of carbon monoxide and peroxyacetyl nitrate on man's maximal aerobic capacity. J Appl Physiol 1974;36:288-93.

[32] Peterson J, Stewart R. Predicting the carboxyhemoglobin levels from carbon monoxide exposures. J Appl Physiol 1975;39:633-9.

[33] Baumann I, Bonov P, Daniels J, et al. NSA round table: high altitude training. New Studies in Athletics 1994;9(2):23-35.

[34] Baker A, Hopkins W. Altitude training for sea-level competition. Sportscience Train Tech. Internet Soc Sport Sci. http://sportsci.org/traintech/altitude/wgh.html, 1998.

[35] Stray-Gundersen J, Chapman R, Levine B. "Living high-training low" altitude training improves sea level performance in male and female elite runners. J Appl Physiol 2001;91(3): $1113-20$. 
[36] Grissom C, Roach R, Sarnquist F, Hackett P. Acetazolamide in the treatment of acute mountain sickness: clinical efficacy and effect on gas exchange. Ann Intern Med 1992;116:461-5.

[37] Keller H, Maggiorini M, Bartsch P, et al. Simulated descent v. dexamethasone in treatment of acute mountain sickness: a randomized trial. BMJ 1995;310:1232-5.

[38] Sophocles A. High altitude pulmonary edema in Vail, Colorado, 1975-1982. West J Med 1986; 144:569-73.

[39] Bartsch P. High altitude pulmonary edema. Med Sci Sport Exerc 1999;31(Suppl 1):S23 - 7.

[40] Durmowicz A, Nooordeweir E, Nicholas R, et al. Inflammatory processes may predispose children to develop high altitude pulmonary edema. J Pediatr 1997;130:838-40.

[41] Hultgren H. High altitude pulmonary edema: hemodynamic aspects. Int J Sports Med (Stuttgart) 1997;18(1):20-5.

[42] Oelz O, Maggiorini M, Ritter M, et al. Prevention and treatment of high altitude pulmonary edema by a calcium channel blocker. Int J Sports Med (Stuttgart) 1992;13(Suppl 1):S65-8.

[43] Cremona G, Asnaghi R, Baderna P, et al. Pulmonary extravascular fluid accumulation in recreational climbers: a prospective study. Lancet 2002;359(9303):303-9.

[44] Campbell E. Decompression illness in sports divers: part I. Medscape Ortho Sports Med e Journal 1997;1(9):1-16.

[45] Schwerzmann M, Seiler C. Recreational scuba diving, patent foramen ovale and their associated risks. Swiss Med Wkly 2001;131(25-26):365-74.

[46] Campbell E. Decompression illness in sports divers: part II. Medscape Ortho Sports Med e Journal 1997;1(9):1-7. 